\title{
Genetic diversity, natural acquired antibody and recombinant vaccine is critical in vaccine malaria disease.
}

\author{
Saeed Nazeri* \\ Malaria and Vector Research Group, Biotechnology Research Center, Pasteur Institute of Iran, No. 69, Pasteur Ave, \\ Tehran, Iran
}

\section{Editorial}

Plasmodium vivax is the most widespread species of human malaria parasites outside Africa that causes malaria morbidity among people of all ages [1] and many people in the world live at risk of $P$. vivax comparing to Plasmodium falciparum [2]. Moreover, malaria control history has displayed that the elimination of $P$. vivax rather than $\mathrm{P}$. falciparum will tend to be technically more challenging, which is in part due to biological characteristics of this parasite species [3]. These characters include the formation of hypnozoites in the liver and gametocytogenesis. Also, there are reports on increasingly severe disease caused by $P$. vivax [4], and emerged chloroquine [5] and multi-drug resistance. Artemisinin combination therapy has less impact on $P$. vivax than on $P$. falciparum [6] and there is currently only one class of drug (8-aminoquinolines) with a known activity against $P$. vivax dormant liver stages. Therefore, there is no doubt that a key tool for the control, elimination, or even possible eradication of malaria in addition to the antimalarial drugs and vector control is the development of a malaria vaccine.

However, antigenic variations limit the immunogenicity of vaccines that signify a major obstruction in the malaria vaccine design. Therefore, to design broadly protective vaccines, it is helpful to have adequate information on the genetic structure of natural $P$. vivax as well as to understand the distribution and natural dynamics of vaccine antigen polymorphisms in global vivax parasite populations.

Since epidemiological and environmental factors, as well as host genetics affect the development of immunity against malaria parasites, detailed profiling of naturally acquired antibodies directed against target parasite antigen in different malaria-endemic regions will provide useful information for vaccine design. $P$. vivax in comparison with $P$. falciparum has been neglected, and so there is a little data on the mechanisms and targets of naturally acquired immunity to this species [7]. Therefore, to battle $P$. vivax during control and elimination campaigns, a greater understanding of $\mathrm{IgG}$ responses to $P$. vivax candidate vaccine antigens in naturally exposed populations is highly required.

On the other hand, the global efforts toward development of a vaccine against $P$. vivax are limited due to the absence of a continuous in vitro culture for $P$. vivax, which has a negative effect on obtaining the large amounts of parasites target antigens for whole vaccine development. Experience has shown that developing vaccines against intracellular pathogens (such as Plasmodium), which require protective Th1 and cell mediate immunity, is more difficult than extracellular pathogens. In addition, in some cases, such as malaria vaccine development, due to the lack of continuous culture for most species of Plasmodium, the only solution could be subunit vaccines; therefore, additional immune stimulants, such as an efficient adjuvant [8-11], is highly important to induce a protective and long-lived immune response [12]. Adjuvant selection may be based on several parameters, including potential use in human, the physical and chemical natures of the vaccine antigen, type of desirable immune response, age of the target population, and the route of vaccine administration. In fact, the selection of an incorrect adjuvant may exhibit a specific vaccine antigen insufficient [13]. Thus, the selection of adjuvant system (AS) is critical to induce proper and protective immune responses, and to increase antibodies with greater avidity, with the potential for neutralization, and with the ability to modulate Th1 cell predominance [14].

\section{References}

1. Cotter C, Sturrock HJ, Hsiang MS, et al. The changing epidemiology of malaria elimination: new strategies for new challenges. Lancet. 2013;382:900-11.

2. Guerra CA, Howes RE, Patil AP, et al. The international limits and population at risk of Plasmodium vivax transmission in 2009. PLoS Negl Trop Dis. 2010;4(8):e774.

3. https://globalhealthsciences.ucsf.edu/pub/shrinkingmalaria-map-guide-malaria-elimination-policy-makers

4. Price RN, Douglas NM, Anstey NM. New developments in Plasmodium vivax malaria: severe disease and the rise of chloroquine resistance. Curr Opin Infect Dis. 2009;22(5):430-5

5. Carrara VI, Sirilak S, Thonglairuam J, et al. Deployment of early diagnosis and mefloquine-artesunate treatment of falciparum malaria in Thailand: the Tak malaria initiative. PLoS Med. 2006;3(6):e183.

6. Mueller I, Galinski MR, Baird JK, et al. Key gaps in the knowledge of Plasmodium vivax, a neglected human malaria parasite. Lancet Infect Dis. 2009;9(9):555-66.

7. Polley SD, McRobert L, Sutherland CJ. Vaccination for vivax malaria: targeting the invaders. Trends Parasitol. 2004;20(3):99-102. 
8. Chitnis CE, Sharma A. Targeting the Plasmodium vivax duffy-binding protein. Trends Parasitol. 2008;24(1):29-34.

9. Herrera S, Corradin G, Arevalo-Herrera M. An update on the search for a Plasmodium vivax vaccine. Trends Parasitol. 2007;23(3):122-8.

10. Remarque EJ, Faber BW, Kocken $\mathrm{CH}$, et al. Apical membrane antigen 1: a malaria vaccine candidate in review. Trends Parasitol. 2008;24(2):74-84.

11. Reed SG, Bertholet S, Coler RN, et al. New horizons in adjuvants for vaccine development. Trends Immunol. 2009;30(1):23-32.

12. Reed SG, Orr MT, Fox CB. Key roles of adjuvants in modern vaccines. Nat Med. 2013;19(12):1597-608.

13. Pashine A, Valiante NM, Ulmer JB. Targeting the innate immune response with improved vaccine adjuvants. Nat Med. 2005;11(4):S63-8.

14. Vogel FR. Improving vaccine performance with adjuvants. Clin Infect Dis. 2000;30(3):S266-70.

\section{*Correspondence to:}

Saeed Nazeri

Malaria and Vector Research Group

Biotechnology Research Center

Pasteur Institute of Iran

No. 69, Pasteur Ave, Tehran, Iran

Email: s.nazeri62@gmail.com 